\title{
1 Bac-PULCE: Bacterial Strain and AMR Profiling Using Long Reads via 2 CRISPR Enrichment
}

\section{Authors}

4 Andrea Sajuthi, Julia White, Gayle Ferguson, Nikki E. Freed*, Olin K. Silander*

5 Address for all authors:

6 School of Natural and Computational Sciences, Massey University, Auckland, New Zealand

7 *Corresponding authors: n.freed@massey.ac.nz (NEF) and olinsilander@gmail.com (OKS)

8

9 Keywords: Nanopore, CRISPR-Cas9, antimicrobial resistance, metagenomics 


\section{Abstract}

11 Rapid identification of bacterial pathogens and their antimicrobial resistance (AMR) profiles is

12 critical for minimising patient morbidity and mortality. While many sequencing methods allow

13 deep genomic and metagenomic profiling of samples, widespread use (for example at

14 point-of-care settings) is impeded because substantial sequencing and computational

15 infrastructure is required for sequencing and analysis. Here we present Bac-PULCE (Bacterial

16 strain and antimicrobial resistance Profiling Using Long reads via CRISPR Enrichment), which

17 combines CRISPR-cas9 based targeted sequence enrichment with long-read sequencing. We

18 show that this method allows simultaneous bacterial strain-level identification and antimicrobial

19 resistance profiling of single isolates or metagenomic samples with minimal sequencing

20 throughput. In contrast to short read sequencing, long read sequencing used in Bac-PULCE

21 enables strain-level resolution even when targeting and sequencing highly conserved genomic

22 regions, such as $16 \mathrm{~S}$ rRNA. We show that these long reads allow sequencing of additional AMR

23 genes linked to the targeted region. Additionally, long reads can be used to identify which

24 species in a metagenomic sample harbour specific AMR loci. The ability to massively multiplex

25 crRNAs suggests that this method has the potential to substantially increase the speed and

26 specificity of pathogen strain identification and AMR profiling, while ensuring low computational

27 overhead. 


\section{Importance}

30 There is a critical need for rapid and identification of bacterial strains and antibiotic resistance

31 profiles in clinical settings. However, most current methods require both substantial laboratory

32 infrastructure (e.g. for DNA sequencing), substantial compute infrastructure (e.g. for

33 bioinformatic analyses), or both. Here we present a new method, Bac-PULCE, (Bacterial strain

34 and antimicrobial resistance Profiling Using Long reads via CRISPR Enrichment), which

35 combines CRISPR-cas9 based targeted sequence enrichment with long-read sequencing on

36 the Oxford Nanopore platform. This allows rapid profiling of bacterial strains and antibiotic

37 resistance genes in a sample while requiring very little laboratory or computational

38 infrastructure. 


\section{Introduction}

40 With the rapid increase in antibiotic resistant bacteria, there is a need for methods to quickly 41 identify antimicrobial resistance (AMR) profiles in clinical samples. Previously, most methods of

42 microbial identification and AMR profiling in clinical practice have been culture based (Andrews,

43 2001; Jorgensen \& Ferraro, 2009; Kiehlbauch et al., 2000), an often slow and laborious process.

44 Over the last decade, a range of newer techniques have been applied for strain and AMR

45 profiling, including whole genome sequencing (Baker et al., 2018), metagenome sequencing

46 (Chiu \& Miller, 2019; Gu et al., 2019), mass spectrometry (Havlicek et al., 2013), microarrays

47 (Wilson et al., 2002), microfluidics (Etayash et al., 2016), and others (Syal et al., 2017). A large

48 number of methods rely on the amplification of specific sequences for diagnosis (Jain et al.,

49 2016; Zumla et al., 2014). However, all of these methods require either substantial infrastructure

50 (e.g. for sequencing and analysis); or are effective in identifying a limited range of bacterial

51 strains or AMR profiles.

52 Recently, Quan et al. 2019 combined CRISPR-based sequence enrichment, PCR, and

53 short-read sequencing to identify bacterial AMR genes in metagenomic samples using a

54 method termed Finding Low Abundance Sequences by Hybridization (FLASH) (Quan et al.,

55 2019). FLASH allows extensive multiplexing for sensitive detection of a wide range of AMR loci

56 while considerably decreasing compute overhead for analysis due to read enrichment. However,

57 there are several limitations of this method. First, the reliance of FLASH on PCR amplification

58 requires enriched loci to be targeted by pairs of crRNAs a specific and relatively short distance

59 apart. Second, the use of short reads makes it difficult to discover linked AMR loci, or AMR

60 context (e.g. plasmid vs. chromosomal). Finally, there are substantial sequencing resources

61 required (although compute resources are considerably reduced).

62 To circumvent these issues, here we present Bacterial strain and antimicrobial resistance

63 Profiling Using Long reads via CRISPR Enrichment (Bac-PULCE), which combines

64 CRISPR-Cas9-based enrichment of conserved bacterial and AMR loci followed by long-read

65 sequencing (Fig S1). We show that this method requires only a single crRNA per target region,

66 greatly increasing flexibility of crRNA design. This method also results in rich information on loci

67 linked to the target sequence of interest, allowing bacterial strain-level resolution even when 


\section{Results}

\section{Enrichment and sequencing of a variable locus in cultured bacteria}

82 To test the feasibility of using CRISPR-Cas9 sequence enrichment and long read sequencing

83 (Profiling Using Long reads via CRISPR Enrichment; Bac-PULCE) for bacterial strain typing, we

84 first designed two crRNAs (see Methods) targeting two sequences surrounding the $E$. coli gnd

85 locus. The gnd locus is known to be highly polymorphic in E. coli, and has been used previously

86 to type strains (Cookson et al., 2017). We designed one crRNA to target a sequence upstream

87 of gnd (within hisF) and the other to target downstream of gnd (within wcaM), with approximately

8820 kilobase pairs (Kbp) between these two target sites (Table 3). We then used the Bac-PULCE

89 method to sequence enriched DNA from E. coli K12 MG1655 on a single MinION flow cell (see

90 Methods).

91 As a result we generated 43,024 reads, totalling 370.3 Megabase pairs (Mbp) of sequence data

92 with a mean read length of $8,606 \mathrm{bp}$. The majority of these reads (52.8\%) mapped to the gnd

93 region. The median coverage depth across the E. coli MG1655 chromosome was 33, while the

94 maximum depth at the cut site in wcaM was 11,799 (Fig 1A). This is an increase of more than

95 350-fold depth at the target site over background. Importantly, we found that the cutting

96 efficiency of each crRNA differed substantially. The his $F$ crRNA was far less efficient at binding 
97 and cutting than the crRNA in wcaM. This is clearly evidenced by examining the number of

98 reads that start at each cut site, with greater than four-fold the number of reads originating at the

99 wcaM cut site compared to hisF (Fig 1B).

100 We also found differences in directionality bias. We expect that the majority of reads starting at a

101 cut site will be in a single direction, despite the fact that the Cas9 cut creates two 5'

102 phosphorylated ends. This is because the CRISPR-Cas9 complex likely remains bound to the

103 strand containing the target site, and prevents motor ligation and sequencing. However, we

104 found that at the hisF cut site, almost equal numbers of reads occurred in both directions (Fig

105 1B). In contrast, at the wcaM locus, a majority of reads started in only one direction. Overall,

106 these results indicated that targeting a locus with a single crRNA should allow efficient

107 enrichment through crRNA binding and cutting, followed by long-read sequencing, although

108 crRNA binding and cutting efficiency can differ considerably. We next tested whether reads at

109 the gnd locus could be used for strain-level identification.

110 We first generated genomic sequence data and assembled the genome of a novel

111 environmental isolate of E. coli strain, L3Cip3 (Van Hamelsveld et al., 2019), into a single

112 circularised $4.93 \mathrm{Mbp}$ chromosomal contig, four circularised contigs likely to be plasmids (177

$113 \mathrm{Kbp}, 88.9 \mathrm{Kbp}, 84.0 \mathrm{Kbp}$, and 44.7 Kbp), and two short circularised contigs likely to be

114 fragments (2255 bp and $1565 \mathrm{bp}$ ) (see Methods). We then mapped the reads generated from

115 Bac-PULCE from the E. coli MG1655 gnd locus to this second strain of E. coli. We found that

116 although these reads mapped, it was readily apparent that they did not map over their full

117 length, as indicated by sudden drops in the coverage depth (Fig. 1C). In this case, the drop in

118 coverage depth was due to the loss (via homologous recombination) of an operon present in $E$.

119 coli MG1655 that contained several genes active in capsule polysaccharide biosynthesis. These

120 results suggested that by using long reads, accurate strain-level classification would be possible

121 using highly variable regions such as the E. coli gnd locus, which is prone to homologous

122 recombination. It also suggested that targeting a more conserved gene, such as $16 \mathrm{~S}$ ribosomal

123 RNA genes would be feasible. 

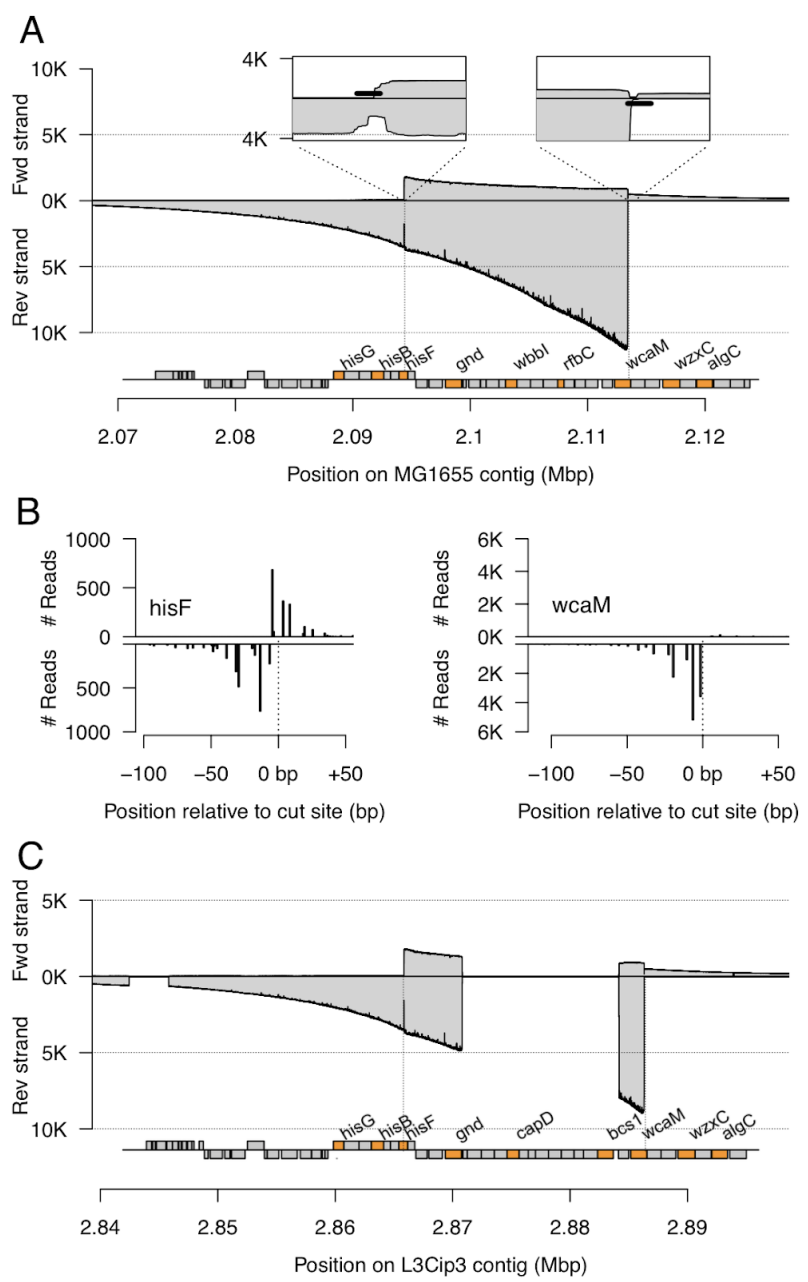

Figure 1. Long reads from Bac-PULCE allow strain-level identification. A. Bac-PULCE allows for more than 100-fold enrichment of sequencing of target loci. We used two crRNAs that flank the highly polymorphic gnd locus in E. coli, one in hisF and the other in wcaM, and sequenced the target-enriched DNA using long read nanopore sequencing. We mapped all reads to the E. coli MG1655 genome. The coverage depth of reads that map to the top strand is shown above the $x$-axis, while the coverage depth of reads mapping to the bottom strand is shown below the x-axis. All annotated genes around the gnd locus are shown beneath, with several genes labelled for context (labelled genes are coloured in orange). The insets show the cut regions at higher resolution, with the binding sites of the crRNAs indicated by thick lines. B. crRNAs exhibit clear differences in efficiency and directionality bias. The two plots indicate the number of reads starting near each crRNA cut site. Lines above the axis indicate reads starting on the top strand; lines below begin on the bottom strand. The left plot indicates the cut site of the hisF crRNA. The right plot indicates the cut site of the wcaM crRNA. The crRNA targeting wcaM is highly efficient and exhibits considerable directionality bias, with almost 10,000 reads originating within $10 \mathrm{bp}$ of the cut site, and these occurring almost solely on the bottom strand. In contrast, the crRNA targeting his $F$ is less efficient, with fewer than 2,000 reads originating within 10 bp of the cut site, and reads starting on both strands. C. Reads from the gnd region of $E$. coli MG1655 mapped to the environmental E. Coli L3Cip3 exhibit substantial gaps due to loss of homology. The $w b b$ operon region has been replaced in L3Cip3 through a homologous recombination event, resulting in a loss of homology. This suggests that strain-level classification may be possible using long reads from the highly variable gnd region. 


\section{Enrichment and sequencing of a conserved locus}

145 The gnd locus is specific to E. coli, and targeting this region in other bacterial taxa, or in a 146 complex metagenomic sample would enrich only for $E$. coli sequences, and thus can not be

147 used to enrich identify sequences from strains of distantly related groups of bacteria. Therefore,

148 we next tested whether accurate strain-level identification would be possible using crRNAs

149 targeting conserved genomic loci. We designed a crRNA targeting the highly conserved 16S

150 ribosomal RNA genes and using Bac-PULCE, enriched and sequenced 16S loci from clonal

151 L3Cip3 genomic DNA.

152 We generated 78,791 reads, with $92.5 \%$ of these reads mapping to the $E$. coli L3Cip3 genome.

153 We also mapped these reads to the E. coli K12 MG1655 genome. We found that even when

154 mapping reads that originated from highly conserved 16S loci (of which there are seven total in

155 E. coli), in the genomic regions surrounding the $16 \mathrm{~S}$ loci, small indels and duplications were

156 present that clearly indicated whether reads had mapped as expected (Fig 2A and B; Fig S2).

157 These could only be observed by relying on long reads that extended beyond the conserved

158165 locus into these more polymorphic regions.

159 To test the limits of taxa identification in a more systematic manner, we mapped the reads

160 originating from the $16 \mathrm{~S}$ loci in L3Cip3 to the rrnDB 16S database (Stoddard et al., 2015), which

161 consists of full length 16S rRNA from 77,530 bacterial species (see Methods). We found that

$16297.6 \%$ of these reads had their primary mapping to ribosomal sequences from either

163 Escherichia or Shigella (which is a polyphyletic genus within the E. coli species complex). The

164 vast majority of incorrect matches were short alignments: $99.8 \%$ of all mappings with alignments

165 longer than 1400bp were to ribosomal sequences from either Escherichia or Shigella. These

166 results suggest that using Bac-PULCE to selectively sequence $16 \mathrm{~S}$ regions allows precise

167 identification of taxa to at least the level of genus. 

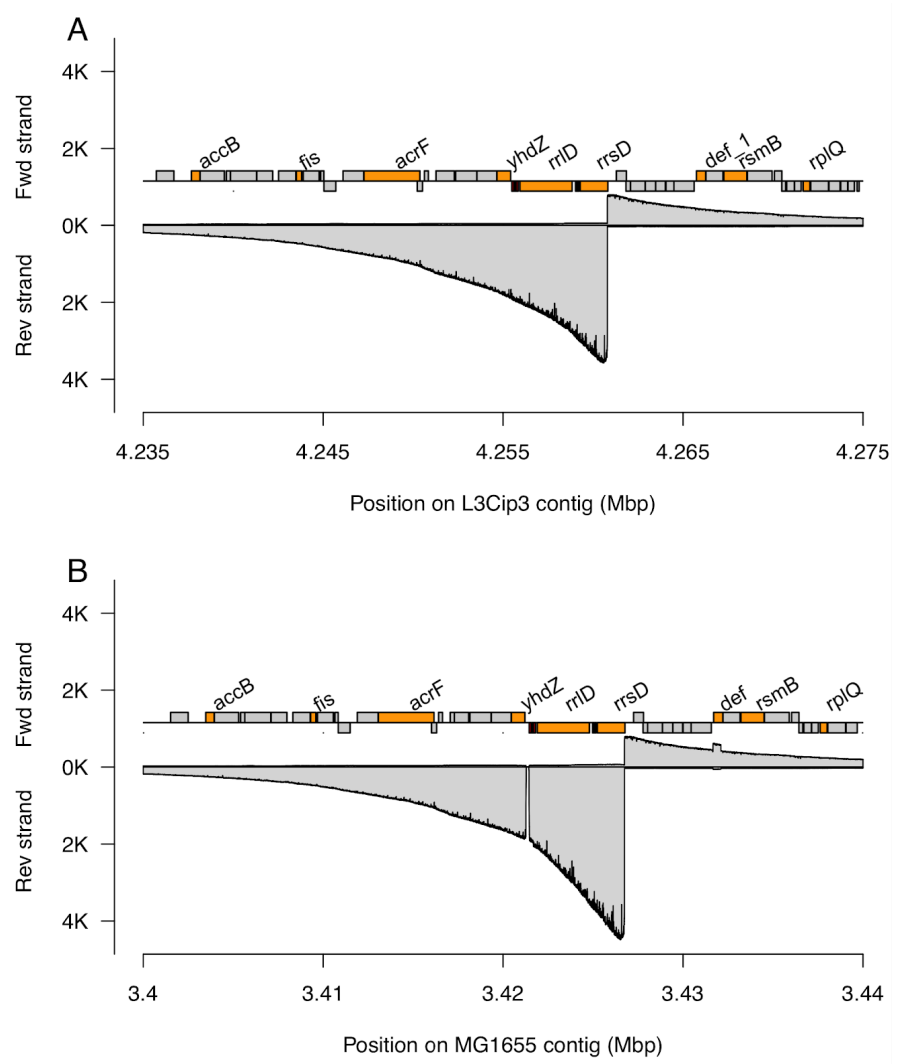

Figure 2. Small insertions and deletions at highly conserved $16 \mathrm{~S}$ loci allow reads from different strains to be distinguished. A. Coverage depth for one of the seven 16S loci in the L3Cip3 genome. The depth of reads that map to the top strand are shown above the x-axis, while reads mapping to the bottom strand are shown below the x-axis. Several ORFs are annotated (coloured in orange). B. Coverage depth for the homologous region in the MG1655 genome. Relative to L3Cip3, there have been small deletions (here in a region just upstream of two tRNAs) and duplications in the MG1655 genome, indicated by drops or increases in coverage. These have occurred adjacent to the highly conserved $16 \mathrm{~S}$ region, but are only apparent with long reads extending beyond the highly conserved $16 \mathrm{~S}$ locus. This locus is one of seven $16 \mathrm{~S}$ loci; all others exhibit similar discrepancies in coverage depth (Fig S2).

\section{Strain level identification}

We next tested the accuracy of using reads from both the gnd and 16S loci for strain-level identification. We mapped the gnd and $16 S$ reads from E. coli L3Cip3 against a database consisting of the L3Cip3 genome and whole genome sequences from 58 additional $E$. coli strains encompassing the diversity of the E. coli clade (see Methods; Fig. S3). For both gnd and 16S, we found that the mapping was highly specific, with approximately $90 \%$ of all $16 \mathrm{~S}$ reads having their primary mapping to the strain of origin. In the case of gnd, this fraction exceeded 99\% (Fig. 3). Furthermore, there was a clear relationship between both mapping 
190 quality and read length on the accuracy of strain-level assignment: long reads and reads with

191 high mapping quality were very likely to correctly identify the strain, with accuracy considerably

192 exceeding $99 \%$ for reads exceeding $15 \mathrm{Kbp}$ in length even for the $16 \mathrm{~S}$ locus. This clearly

193 indicates that even when using Bac-PULCE to target highly conserved loci such as 16S rRNA

194 genes, it is possible to precisely identify the bacteria at the strain-level. This vastly improves

195 taxonomic resolution beyond what is currently possible when sequencing just the 16S region, and is

196 made possible by the length of the reads (Johnson et al., 2019).
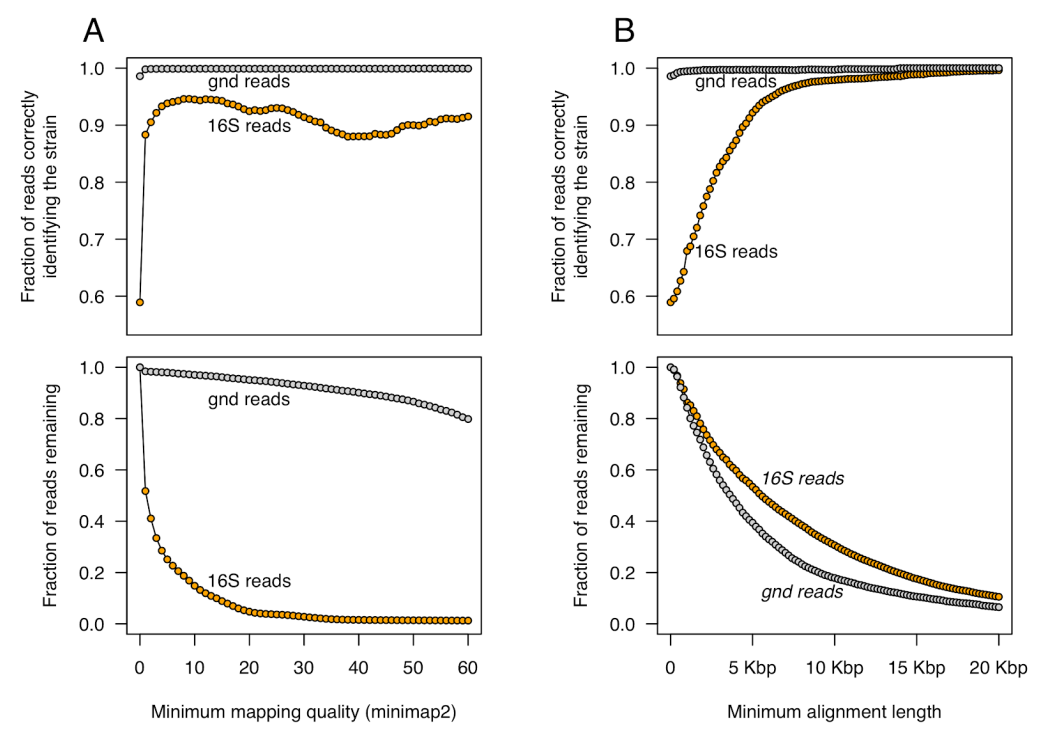

\section{Figure 3. Long reads allow unambiguous identification of $E$. coli taxa at the strain level. A.} Relationship between mapping quality and the accuracy of strain assignment for the gnd and 16S loci. The top panel shows the fraction of reads mapped to the correct strain (L3Cip3) as a function of mapping quality, while the bottom panel shows the fraction of reads with that mapping quality or higher. At a minimum mapping quality of 1 almost $90 \%$ of all 16 S reads map to the correct strain despite this locus being highly conserved. The fraction or correctly mapped reads is far higher for the polymorphic gnd locus. B. Relationship between read length and the accuracy of strain assignment. The top panel shows the fraction of reads mapped to the correct strain (L3Cip3) as a function of read length, while the bottom panel shows the fraction of reads of that read length or longer. In contrast to the relationship between read quality and classification accuracy for $16 \mathrm{~S}$, at long read lengths (e.g. more than $15 \mathrm{Kbp}$ ), the accuracy of strain assignment exceeds $99 \%$. 


\section{Sequence context affects Bac-PULCE efficiency}

211 The data here show that accurate strain-level classification is possible even when targeting

212 highly conserved loci. In addition, we found that binding and cutting efficiency can differ

213 substantially between loci. We hypothesised that these differences could arise either from the

214 specific target sequence, or from the genomic context of the target site. We thus next examined

215 variability in the cutting efficiency of different loci for an individual crRNA (Liu et al., 2016).

216 We designed a crRNA targeting identical sequences in multiple copies of beta-lactamase genes

217 which were present on two plasmids in L3Cip3. All three of these copies are identical in

218 sequence. Here again we found that the crRNA cut with high efficiency and specificity, but that

219 this varied among cut sites both in terms of strand bias and efficiency (Fig. 4A and 4B), despite

220 the target sequences being identical. Of the three beta-lactamase loci targeted by the crRNA,

221 one cut such that Oxford Nanopore motors ligated to both strands at almost equal levels (Fig

222 4C), while a second cut such that reads were phosphorylated almost exclusively at only one

223 end. Again, we hypothesise that directionality bias is due the CRISPR-Cas9 complex remaining

224 bound to the DNA and subsequently blocking ligation of the motor complex. However, in contrast

225 to the results above, when we repeated this analysis for the crRNA targeting the 16S regions,

226 we found that all seven 16 S regions were cut and sequenced almost identically (Fig. S4).

227 Bac-PULCE allows identification of additional linked AMR loci

228 In addition to long reads providing information on polymorphic regions near to conserved 16S

229 loci, long reads allowed ready identification of additional AMR loci linked to the targeted

230 bla-TEM loci. One plasmid had only a single copy of the bla-TEM locus, and was thus cut once

231 (Fig 4B). However, the majority of the reads extended well beyond this locus, such that an

232 additional three AMR loci were sequenced, including a gene for aminoglycoside resistance,

233 dihydrofolate resistance, and tetracycline resistance. The maximum coverage depth on the

234 bottom strand of the targeted bla-TEM locus on this plasmid at position $81.1 \mathrm{Kbp}$ was 2,488 .

235 Median read depth was 2,028 at the aminoglycoside resistance locus $1.9 \mathrm{Kbp}$ upstream of the

236 targeted locus; 1,028 at the dihydrofolate resistance locus $9.1 \mathrm{Kbp}$ upstream; and 564 at the

237 tetracycline resistance locus $16.8 \mathrm{Kbp}$ upstream. This contrasts with a median depth of 67 over

238 the whole plasmid. This is also apparent at the level of individual reads. 3,049 reads begin or

239 end within the targeted bla-TEM gene (the majority on the bottom strand). 2,113 (69.3\%) of 


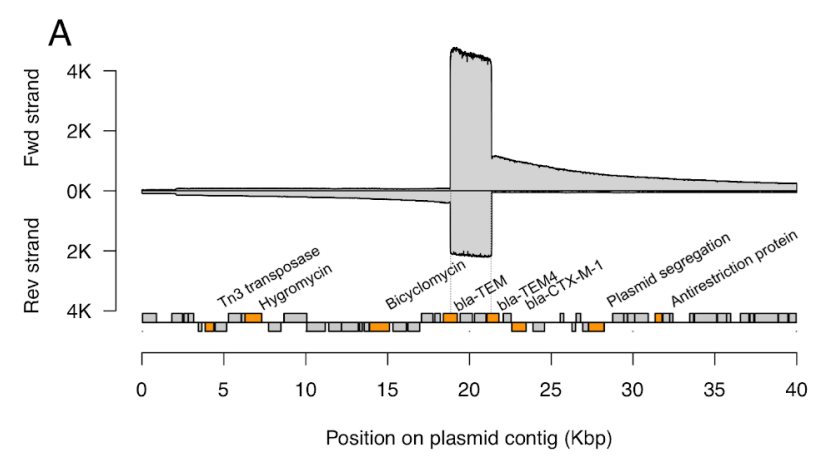

B
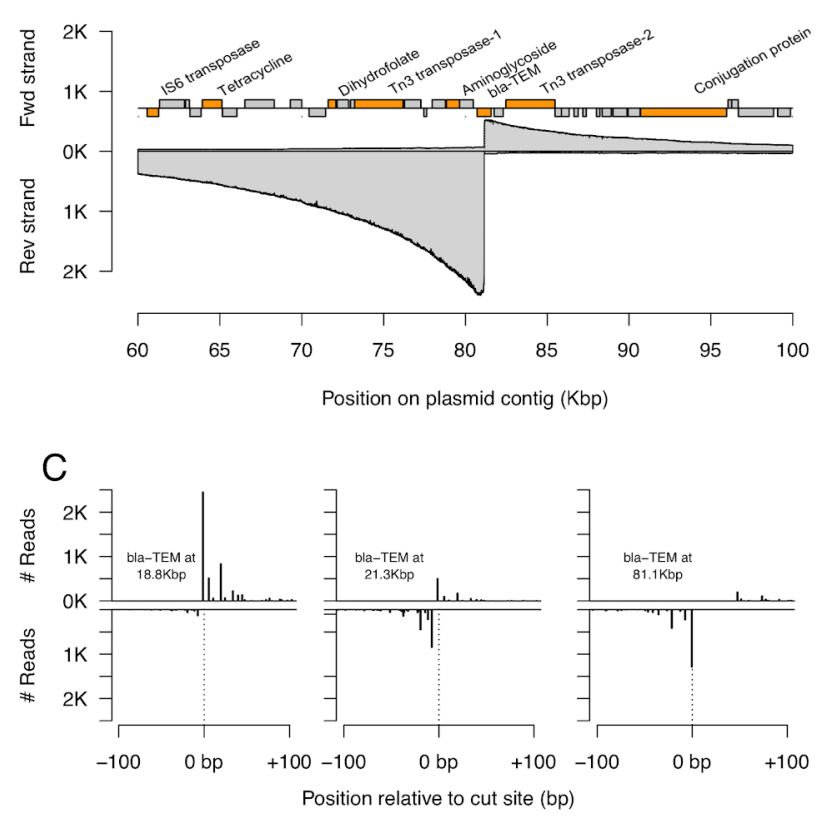

240 Figure 4. Variability in crRNA cutting for identical target sequences. We used a single crRNA to target multiple versions of a beta-lactamase resistance gene present on two different plasmids in L3Cip3. These target loci have identical sequences, although the surrounding sequence context is different. A. Coverage depth at a region with two beta-lactamase genes and target cut sites. The depth of reads that map to the top strand are shown above the x-axis, while reads mapping to the bottom strand are shown below the x-axis, with several ORFs annotated (coloured in orange), including the beta-lactamase resistance genes (bla-TEM). B. Coverage depth at a region with a single beta-lactamase gene and target cut site. $C$. Each cut site exhibits a unique binding and cutting efficiency as well as directionality bias. The three plots indicate the number of reads starting near each crRNA cut site. Lines above the axis indicate reads starting on the top strand; lines below begin on the bottom strand. The plots are shown in the order of cut sites, with the locations of the cut sites indicated on each plot. Cuts at the first bla-TEM locus are efficient and have a clear directionality bias; cuts at the second bla-TEM locus are less efficient and have less bias, with reads almost equally likely to start on the top or bottom strand. Reads at the third bla-TEM locus again show clear bias. This locus is on a separate plasmid that is present at approximately 0.41 lower copies than the first (as inferred through read coverage), suggesting

255 that cutting efficiency differs little between the first and third cut sites. 
257 these include part or all of the aminoglycoside locus; 981 (32.2\%) contain part or all of the 258 dihydrofolate locus; and 530 (17.4\%) contain part or all of the dihydrofolate locus.

\section{Sensitivity and multiplexing capability}

260 Finally, we tested the sensitivity and multiplexing capability of this method. We first sequenced a

261 metagenomic sample consisting of faecal samples from four sheep and one cow on a single

262 MinION flow cell (see Methods), yielding a total of 8.83 million reads and $24.5 \mathrm{Gbp}$, an amount

263 of data that required more than 24 hours to basecall on standard GPU, and far longer using

264 CPU resources alone. We mapped these reads against the resfinder database (Bortolaia et al.,

$2652020)$ to identify AMR loci. We found a total of 188 reads matching AMR loci $(0.002 \%$ of all

266 reads). This varied substantially between samples, from $0.0052 \%$ in the single cow sample (124

267 out of 2.36 million reads) to $0.00071 \%$ (5 out of 702 thousand reads) in one sheep sample.

268 We next designed crRNAs targeting ten different AMR loci found in this metagenomic sample

269 (see Methods). Pooling several faecal samples together, we used these ten crRNA and an

270 additional crRNA targeting 16S, and performed Bac-PULCE using a single MinION flow cell.

271 This resulted in a total of 37,200 reads. Of these, 53 reads $(0.14 \%)$ mapped to four different

272 AMR types (Table 1). Some of these were sequenced in numbers close to that of the original

273 metagenomic run (e.g. $c f x A$ ), despite sequencing approximately 250 -fold less data in the

274 Bac-PULCE run. This clearly illustrates the power of this approach, in that far less data is

275 required to achieve a similar level of accuracy in AMR profiling. However, other AMR loci were

276 sequenced far less efficiently or not at all (e.g. the ResFinder loci aac(6')-aph(2")_1_M13771 or

277 aph(2")-la_2_AP009486, which provide aminoglycoside resistance).

278 We next aimed to identify the organismal context of these AMR loci, relying on the length of the

279 reads to provide this context. Focusing only on the individual reads that mapped to cfxA genes

280 in ResFinder, we used BLAST to find matching taxa in the nt database (see Methods), only

281 considering reads with more than $100 \mathrm{bp}$ of sequence that was not part of the cfxA gene (36 out

282 of 39 reads). We found that the majority of cfxA genes were contained in a chromosomal

283 context in Prevotella spp. (54\%; Table 2), despite Prevotella spp. being present at less than $1 \%$ 
284 frequency across all samples. Thus, leveraging read length yields considerable insight into the

285 organismal and genomic context of these AMR loci.

\section{6}

287 Table 1. Number of reads found for different AMR loci. The locus (as annotated in ResFinder) is 288 indicated in the first column, with two exceptions, cfxA and tet(W), for which the specific AMR types

289 cannot be differentiated because the crRNA targets a conserved region in the locus. The number of reads

290 mapping to each locus are indicated in the second and third columns for the full metagenomic run and

291 Bac-PULCE run, respectively, while the last column notes the fold-enrichment of the locus in the

292 Bac-PULCE run.

\begin{tabular}{lllc} 
AMR locus & $\begin{array}{l}\text { Total metagenomic } \\
\text { Reads }(\%)\end{array}$ & $\begin{array}{l}\text { Total Bac-PULCE } \\
\text { reads }(\%)\end{array}$ & $\begin{array}{l}\text { Fold-enrichment } \\
\text { in Bac-PULCE }\end{array}$ \\
\hline cfxA & $80(0.0009 \%)$ & $39(0.1 \%)$ & 116 \\
\hline Inu(C)_1_AY928180 & $11(0.0001 \%)$ & $11(0.03 \%)$ & 237 \\
\hline catB_1_M93113 & $7(0.00008 \%)$ & $1(0.003 \%)$ & 34 \\
\hline tet(W) & $3(0.00003 \%)$ & $2(0.005 \%)$ & 158 \\
\hline aac(6')-aph(2")_1_M13771 & $10(0.0001 \%)$ & 0 & NA \\
\hline aph(2")-la_2_AP009486 & $9(0.0001 \%)$ & 0 & NA \\
\hline nimJ_1_NZ_JH815495 & $2(0.00003 \%)$ & 0 & NA \\
\hline tet(O)_1_M18896 & $2(0.00003 \%)$ & 0 & NA
\end{tabular}

295 Finally, we quantified the efficiency of 16 S enrichment from the metagenomic sample. We first

296 mapped all reads from the full metagenomic run to the rnDB 165 database. For the full

297 metagenomic run, 61,779 reads (0.70\%) mapped to this database. As alignment length is

298 closely related to the accuracy of taxon matches, we filtered this set to consider only read

299 alignments longer than 1200bp (near full length 16S matches (Cuscó et al., 2017). This resulted

300 in 17,257 reads (0.20\%). In the same Bac-PULCE run as above, we obtained 1,127 reads

301 (3.0\%) matching the $16 \mathrm{~S}$ rrnDB, with 692 (1.9\%) of these reads being longer than $1200 \mathrm{bp}$. This

302 is only $4 \%$ of the total full length alignments we obtained in the metagenomic run, and suggests

303 that although $16 \mathrm{~S}$ regions were enriched in this dataset, the efficiency was far below the

304 enrichment for AMR loci. 
306 Table 2. Organismal context of cfxA resistance loci. We trimmed all Bac-PULCE reads mapping to cfXA genes to remove the portion matching the cfxA gene, and BLASTed the read against the NCBI nt database. The genus of the top hit is listed in the first column, followed by the community prevalence of that taxa (inferred through mapping $16 \mathrm{~S}$ reads to $\mathrm{rnDB}$ ), followed by the number of cfxA-linked reads mapping to that genus. Several genera are overrepresented in cfxA-linked reads compared to what would be expected from their community prevalence. The last column indicates the median percent identity for all cfxA-linked reads mapping to that genus. Oxford Nanopore reads have a mean accuracy of approximately $93 \%$, so we would expect a strain-level match to be approximately $93 \%$ identical and a 314 species-level match to be slightly lower. Most matches have $90 \%$ or less identity; thus we identify taxa 315 only at the level of genus.

\begin{tabular}{llll} 
Genus & $\begin{array}{l}\% \text { of bacterial } \\
\text { community }\end{array}$ & $\begin{array}{l}\% \text { of cfxA-linked } \\
\text { reads (number) }\end{array}$ & $\begin{array}{l}\text { median \% identity of } \\
\text { cfxA-linked reads }\end{array}$ \\
\hline Prevotella & $0.78 \%$ & $54 \%(21)$ & $90.0 \%$ \\
\hline Porphyromonas & $0.12 \%$ & $8 \%(3)$ & $81.0 \%$ \\
\hline Tannerella & $0.40 \%$ & $8 \%(3)$ & $89.0 \%$ \\
\hline Bacteroides & $6.98 \%$ & $5 \%(2)$ & $80.9 \%$ \\
\hline Capnocytophaga & $0.05 \%$ & $5 \%(2)$ & $91.3 \%$ \\
\hline Lachnospiraceae & $2.88 \%$ & $5 \%(2)$ & $96.4 \%$ \\
\hline Chryseobacterium & $0.02 \%$ & $2.6 \%(1)$ & $96.4 \%$ \\
\hline
\end{tabular}

\section{Discussion}

318 Here we have shown that by targeting and enriching specific loci using CRISPR-cas9 to cut at a 319 single locus, followed by long-read sequencing (Bac-PULCE), we can profile bacterial taxa at 320 strain-level accuracy. We have shown this is possible using highly conserved 16S rRNA loci, 321 allowing for far greater taxonomic resolution than is currently available from even sequencing 322 the full length $16 \mathrm{~S}$ gene. We have also shown this method is able to target and enrich, by over

323 100-fold, sequences from AMR loci in a complex metagenomic sample. Additionally, the long 324 reads generated from Bac-PULCE allow sequencing of unknown loci (e.g. additional AMR 325 genes) linked to targeted regions.

326 We found wide variation in the efficiency with which different targets were bound and cut by the 327 crRNA. This was most clear when using Bac-PULCE for enrichment of AMR loci from the 328 metagenomic sample: we failed to sequence some AMR loci at all, although up to ten reads 329 were sequenced during the full metagenomic run. In addition to this probable crRNA 330 sequence-dependence, we found that the efficiency of target enrichment depends on the larger 
sequence context of the crRNA binding site: identical sequences in different genomic locations can differ by more than two-fold in efficiency. The variability we observed emphasises the necessity of optimising crRNA pools for efficient binding, cas9 cutting, and sequencing. This is best illustrated by the inefficient enrichment of $16 \mathrm{~S}$ loci from metagenomic samples that we observed: despite observing more than 300-fold enrichment of $16 \mathrm{~S}$ loci in single isolates, we observed only 10 -fold enrichment from the metagenomic sample. Further work using large scale multiplexing in complex samples should allow the optimization of crRNA target sites to improve the efficiency of the Bac-PULCE approach.

There are three primary advantages of Bac-PULCE over other CRISPR-Cas9 enrichment strategies and short-read sequencing methods such as FLASH (Quan et al., 2019). First, by targeting AMR loci with single crRNAs, long reads enable sequencing of linked AMR loci, increasing the resolution of profiling even when all AMR genes are not targeted for enrichment and sequencing. This is also advantageous for profiling bacterial strains: highly conserved loci, such as 16S, can be targeted such that a broad range of bacteria can be profiled. By matching these sequences against $16 \mathrm{~S}$ databases (such as rnDB), major genera can be profiled. Strain-level resolution can then be obtained by taking the subset of reads that match each genus (or species), and mapping these against genomes from a wide range of strains within this genus (as we have done here). This is a powerful approach, and could allow strain-level resolution of pathogens from complex samples even when the genus or family of the pathogen is unknown.

Second, because sequencing reads can be of any length and no PCR step is used, only a single cut site is required, considerably increasing flexibility. Third, very little sequencing throughput is required for successful strain typing and AMR profiling. This is critical because although Oxford Nanopore sequencing requires very little laboratory infrastructure, there are still considerable demands for compute power. For example, basecalling a single run usually requires more than 24 hours on a standard GPU. Downstream bioinformatic analyses require additional compute power. Thus, we expect that the limited sequencing throughput required for successful strain typing and AMR profiling should allow rapid screening of complex samples using low-cost infrastructure and less than 1/100th of the compute resources for both DNA sequencing and downstream analyses. 
361 There are, however, two drawbacks to the Bac-PULCE approach at this point. The first is that it

362 requires substantial biomass. Here we have used samples from pure culture or from fecal

363 samples, yielding $\mu \mathrm{g}$ quantities of DNA. This requirement contrasts with approaches that rely

364 upon enrichment followed by amplification, such as FLASH (Quan et al., 2019). However, we

365 expect that by combining Bac-PULCE with methods of non-specific DNA amplification, such as

366 those used for whole genome amplification, we may be able to considerably decrease the

367 amount of biomass required. Second, sequencing efficiency is low. This is a function of both the

368 rarity of the target sequences in the sample, and the efficiency of crRNA binding, cas 9 cutting,

369 and attachment of the motor protein. Again, we expect that we can exploit the flexibility of

370 requiring only a single cut site, and the possibility of using highly multiplexed pools of crRNAs to

371 select the most efficient crRNAs for each target sequence of interest (e.g. 16S rRNA). This

372 should further increase the sequencing efficiency and throughput of this approach.

\section{Abbreviations}

374 CRISPR - clustered regularly interspaced short palindromic repeats; crRNA - CRISPR RNA;

375 Bac-PULCE - Bacterial strain and antimicrobial resistance Profiling Using Long reads via

376 CRISPR Enrichment; AMR - antimicrobial resistance; FLASH - Finding Low Abundance

377 Sequences by Hybridization; Mbp - megabase pairs; Kbp - kilobase pairs; bla-TEM - TEM beta

378 lactamase; GPU - graphics processing unit 


\section{Methods}

\section{DNA isolation}

381 We isolated bacterial genomic DNA from $2 \mathrm{~mL}$ of overnight cultures using the Promega Wizard

382 Genomic DNA Purification Kit per manufacturer instructions with the following modifications.

383 Following the protein precipitation step, we performed an additional centrifugation step.

384 Additionally, we washed the DNA pellet twice in $70 \%$ ethanol. We rehydrated the DNA in $32 \mathrm{uL}$

385 water overnight for 18 hours.

386 DNA from cow and sheep faecal samples was extracted using the Qiagen PowerSoilPro kit 387 according to manufacturer instructions.

\section{Genome sequencing}

389 For Nanopore bacterial genome sequencing of L3Cip3 (Van Hamelsveld et al., 2019), we

390 followed the manufacturer's protocol for the SQK-RBK004 kit (Version:

391 RBK_9054_v2_revM_14Aug2019). We sequenced the sample on a R9.4 flow cell (MinION

392 software MinKnow 3.6.0) and basecalled using guppy v3.4.4. Illumina sequencing was

393 performed by the Microbial Genome Sequencing (MiGS) Center using 150bp PE reads.

\section{Genome assembly}

395 We used Unicycler v0.4.5 (Wick et al., 2017) for hybrid genome assembly of L3Cip3, with a total 396 of $221 \mathrm{Mbp}$ of Oxford Nanopore data (mean length $2.3 \mathrm{Kbp}$ ) and 150bp PE Illumina data (1.99M

397 reads, 525.6 Mbp). We annotated the assembly using prokka v1.14.6 (Seemann, 2014).

\section{8 crRNA design}

399 To enrich for the gnd locus we targeted conserved sequences in the hisF and wcaM open

400 reading frames. To enrich for $16 \mathrm{~S}$ loci we targeted a sequence in $\mathrm{rrs}$, which is present in all

401 seven E. coli ribosomal operons. To enrich for beta-lactamase AMR we designed a crRNA that 
402 matched all three bla-TEM loci in L3Cip3. To design crRNAs targeting all other AMRs, we used

403 the sequences of the AMR locus found in the ResFinder 4.0 database (Bortolaia et al., 2020).

404 To design crRNA targeting gnd, beta-lactamase, and 16S, we used CHOPCHOP with the

405 CRISPR/Cas9 setting (Labun et al., 2016, 2019), using the human GRCh38 as background. For

406 all other crRNAs, we used the same settings except with Bos taurus as background. We set

407 sgRNA length without PAM as 20, PAM-3' as NGG, allowed up to 3 mismatches in the

408 protospacer, and used the efficiency score from Doench et. al. 2014 (Doench et al., 2014). We

409 filtered all results to retain sequences with GC content between 40-80\%, self-complementarity

410 scores of 0 , Mismatch (MM) 1 scores of 0 , MM2 scores of 0 , and MM3 scores $<5$.

\section{1 crRNA and tracrRNA synthesis}

412 We in vitro transcribed crRNA and tracrRNA from DNA oligos using a modified in vitro

413 transcription protocol (Quan et al., 2019). Briefly, to all crRNA sequences (Table 3) we added

414 the T7 RNA polymerase binding site (5'-TAATACGACTCACTATAG-3') at the 5' end. To the 3' end

415 of the crRNA sequences, we added the tracrRNA binding sequence

416 (5'-GTTTTAGAGCTATGCTGTTTTG-3') to allow base-pairing of the crRNA to the tracrRNA.

417 To transcribe the tracrRNA, we used a DNA oligo with the full length tracrRNA sequence

418 together with T7 RNA polymerase binding site at the 5' end (underlined). Nucleotides in bold are

419 positions that form base-pairing between the tracrRNA binding sequence and the full length

420 tracrRNA.

\section{TAATACGACTCACTATAGGACAGCATAGCAAGTTAAAATAAGGCTAGTCCGTTATCAACTTGA}

\section{AAAAGTGGCACCGAGTCGGTGCTTTTT 3`}

423 To transcribe the crRNA and tracrRNA from DNA oligos, we used the in vitro transcription

424 protocol from Lyden et al. 2019 (Lyden, 2019). up to the step of RNA synthesis. For RNA

425 synthesis we used the NEB Standard RNA Synthesis protocol (E2050, New England Biolabs).

426 We then added $1.5 x$ volumes of ethanol to the reaction, followed by purification using a $1 x$

427 volume of Ampure XP beads. We eluted the RNA off the beads in $32 \mu \mathrm{L}$ water.

428 Table 3. crRNA sequences. The locus (as named in ResFinder for the AMR loci; or the named 429 locus for E. coli MG1655) is listed in the first column, and the 5' to 3' sequence of the crRNA is 
430 listed in the second column. All target regions matching the crRNA have a NGG PAM sequence

431 at the 3 ' end.

\begin{tabular}{ll} 
Target locus & crRNA sequence \\
\hline aac(6')-aph(2")_1_M13771 & AUUGGUGCAAUCCCUCAAUA \\
\hline aph(2")-la_2_AP009486 & CCAGAACAUGAAUUACACGA \\
\hline blaOXA-235_1_JQ820240 & ACGUGCCAGUUCCUGAUAGA \\
\hline catQ_1_M55620 & AAUCCGGUAAAAUUCACCCA \\
\hline cfxA4_1_AY769933 & ACCGCCACACCAAUUUCGCC \\
\hline Inu(C)_1_AY928180 & CAUCAAACUCGUAUCCCAGA \\
\hline mef(A)_1_AJ971089 & CUUUCGGUGCCAUUUUAUAG \\
\hline nimJ_1_NZ_JH815495 & UAUGACCGCUCAGUGCACUA \\
\hline tet(O)_1_M18896 & AAGCCUGCUCCAAUACGAUA \\
\hline tet(W)_1_DQ060146 & ACGCUGCCGCUCCAAAAACA \\
\hline rrsH & UGGCUCAGAUUGAACGCUGG \\
\hline wcaM & AAUUACGCCAUCUUACGCCA \\
\hline hisF & GUACAGGAAGUGCAAAAACG \\
\hline beta-lactamase (L3Cip3) & UUACUUCUGACAACGAUCGG
\end{tabular}

\section{CRISPR-Cas9 enrichment and sequencing}

434 For target sequence enrichment we used the Oxford Nanopore Cas-mediated PCR-free

435 enrichment protocol v. ENR_9084_v109_revF_04Dec2018 per manufacturer instructions.

436 Briefly, we prepared ribonuclear proteins (RNPs) using pooled crRNAs, tracrRNA, and

437 Integrated DNA Technologies Alt-R S.p. HiFi Cas9 Nuclease V3. We then combined

438 dephosphorylated DNA samples with the RNPs. We dA tailed the CRISPR-Cas 9 cleaved target

439 sequences and ligated adapters to these ends.

\section{Basecalling and demultiplexing}

441 For basecalling and demultiplexing we used three versions of the Oxford Nanopore guppy

442 basecaller: v.3.2.6 (for the experiment using crRNA targeting wcaM and hisF); v.3.4.4 (for the

443 experiments targeting wcaM,16S, and beta-lactamase; and for the full metagenomic 
444 sequencing); or v.4.0.14 (for the experiment using Bac-PULCE on metagenomic DNA sample).

445 These versions differ by approximately $1 \%$ in mean accuracy, and we do not expect that this 446 affects our results here.

\section{Read mapping and analysis}

448 For all read mapping we used minimap2 with the flags map-ont and --secondary=no. To test the 449 specificity of mapping for reads originating from the MG1655 gnd locus, we considered only 450 reads mapping to a $100 \mathrm{Kbp}$ region surrounding the gnd locus in MG1655. To test the specificity 451 of mapping for reads originating from $16 \mathrm{~S}$ loci, we first extracted reads containing any partial 45216 Sequence by mapping all reads against all rrnDB sequences from Escherichia or Shigella.

453 To test for genus-level specificity we then mapped this subset of $16 \mathrm{~S}$ reads from the sample to 454 the full rrnDB database. To test for strain-level specificity, we mapped the read subsets to a 455 database consisting of 58 whole genomes of E. coli (Breckell \& Silander, 2020).

456 To calculate the number of reads originating at the bla-TEM locus that also contained the 457 upstream aminoglycoside, dihydrofolate, or tetracycline AMR loci, we extracted all reads 458 originating within the bla-TEM locus, and mapped these to the open reading frames of the 459 respective AMR gene using minimap2. We inferred that reads successfully mapping to these 460 ORFs contained enough information to determine whether that AMR gene was also present on 461 the read, and thus co-occuring with the targeted AMR locus (in this case, bla-TEM).

462 To infer bacterial taxa present in the cow and sheep metagenomic samples using $16 \mathrm{~S}$ reads, we 463 mapped all reads to the $16 \mathrm{~S}$ rnDB database. We then filtered all matches to consider only 464 near-full length matches (more than 1200 bp).

465 To infer the organismal context of the cfxA loci in this complex metagenomic sample, we first 466 identified the reads mapping to any cfxA genes in ResFinder. We then trimmed the portion of 467 the read matching the gene, plus approximately 30 additional $\mathrm{bp}$, and only retained reads with 468 more than $100 \mathrm{bp}$ of trimmed sequence. We then BLASTed the remaining portion of each read 469 against a local nt database (downloaded on November 1, 2019).

470 We performed all statistical analyses using $R$ v 4.0.2 (Stoddard et al., 2015). We performed all 471 visualisations of genomic loci using genoplotR (Guy et al., 2010). 


\section{Availability of data and materials}

473 All read data are available from NCBI (BioProject PRJNA665129). The genome sequence of

474 L3Cip3 is available as BioSample SAMN16242922.

\section{Competing interests}

476 The authors declare that they have no competing interests.

\section{Funding}

478 This work was funded through a Marsden grant (MAU-1703) to OS and a Massey University

479 Research Fund grant to NF. The funders had no role in the design, analysis, or interpretation of

480 data.

\section{Authors' contributions}

482 OKS and NEF conceived and designed the experiments. AS, JW, GF, and NEF performed the

483 experiments. OKS performed the computational analyses. OKS and AS drafted the manuscript, 484 with input from NEF. All authors read and approved the manuscript.

\section{Acknowledgments}

486 We thank the Heinemann group at the University of Canterbury for providing the L3Cip3 isolate

487 and Dr. Megan Devane of the Environmental Science and Research Crown Research Institute

488 of New Zealand for providing faecal material for metagenomic sequencing. 


\section{References}

513 Cuscó, A., Viñes, J., D’Andreano, S., Riva, F., Casellas, J., Sánchez, A., \& Francino, O. (2017).

Andrews, J. M. (2001). Determination of minimum inhibitory concentrations. The Journal of Antimicrobial Chemotherapy, 48 Suppl 1, 5-16. https://doi.org/10.1093/jac/48.suppl_1.5

Baker, S., Thomson, N., Weill, F.-X., \& Holt, K. E. (2018). Genomic insights into the emergence and spread of antimicrobial-resistant bacterial pathogens. Science (New York, N.Y.), 360(6390), 733-738. https://doi.org/10.1126/science.aar3777

Bertels, F., Silander, O. K., Pachkov, M., Rainey, P. B., \& van Nimwegen, E. (2014). Automated reconstruction of whole-genome phylogenies from short-sequence reads. Molecular Biology and Evolution, 31(5), 1077-1088. https://doi.org/10.1093/molbev/msu088

Bortolaia, V., Kaas, R. S., Ruppe, E., Roberts, M. C., Schwarz, S., Cattoir, V., Philippon, A., Allesoe, R. L., Rebelo, A. R., Florensa, A. F., Fagelhauer, L., Chakraborty, T., Neumann, B., Werner, G., Bender, J. K., Stingl, K., Nguyen, M., Coppens, J., Xavier, B. B., ... Aarestrup, F. M. (2020). ResFinder 4.0 for predictions of phenotypes from genotypes. The Journal of Antimicrobial Chemotherapy. https://doi.org/10.1093/jac/dkaa345

Breckell, G., \& Silander, O. K. (2020). Complete Genome Sequences of 47 Environmental Isolates of Escherichia coli. Microbiology Resource Announcements, 9(38). https://doi.org/10.1128/MRA.00222-20

Chiu, C. Y., \& Miller, S. A. (2019). Clinical metagenomics. Nature Reviews. Genetics, 20(6), 341-355. https://doi.org/10.1038/s41576-019-0113-7

Cookson, A. L., Biggs, P. J., Marshall, J. C., Reynolds, A., Collis, R. M., French, N. P., \& Brightwell, G. (2017). Culture independent analysis using gnd as a target gene to assess Escherichia coli diversity and community structure. Scientific Reports, 7(1), 841. https://doi.org/10.1038/s41598-017-00890-6 Using Min/ON ${ }^{T M}$ to characterize dog skin microbiota through full-length 16S rRNA gene sequencing approach. 20. https://doi.org/10.1101/167015 

for CRISPR-Cas9-mediated gene inactivation. Nature Biotechnology, 32(12), 1262-1267. https://doi.org/10.1038/nbt.3026

Etayash, H., Khan, M. F., Kaur, K., \& Thundat, T. (2016). Microfluidic cantilever detects bacteria and measures their susceptibility to antibiotics in small confined volumes. Nature Communications, 7(1), 12947. https://doi.org/10.1038/ncomms12947

FigTree. http://tree.bio.ed.ac.uk/software/figtree/

527

Gu, W., Miller, S., \& Chiu, C. Y. (2019). Clinical Metagenomic Next-Generation Sequencing for Pathogen Detection. Annual Review of Pathology, 14, 319-338. https://doi.org/10.1146/annurev-pathmechdis-012418-012751

Guy, L., Roat Kultima, J., \& Andersson, S. G. E. (2010). genoPlotR: Comparative gene and genome visualization in R. Bioinformatics, 26(18), 2334-2335. https://doi.org/10.1093/bioinformatics/btq413

Havlicek, V., Lemr, K., \& Schug, K. A. (2013). Current trends in microbial diagnostics based on mass spectrometry. Analytical Chemistry, 85(2), 790-797. https://doi.org/10.1021/ac3031866

Jain, M., Olsen, H. E., Paten, B., \& Akeson, M. (2016). The Oxford Nanopore MinION: Delivery of nanopore sequencing to the genomics community. Genome Biology, 17(1), 239. https://doi.org/10.1186/s13059-016-1103-0

Johnson, J. S., Spakowicz, D. J., Hong, B.-Y., Petersen, L. M., Demkowicz, P., Chen, L., Leopold, S. R., Hanson, B. M., Agresta, H. O., Gerstein, M., Sodergren, E., \& Weinstock, G. M. (2019). Evaluation of $16 \mathrm{~S}$ rRNA gene sequencing for species and strain-level microbiome analysis. Nature Communications, 10(1), 5029. https://doi.org/10.1038/s41467-019-13036-1

Jorgensen, J. H., \& Ferraro, M. J. (2009). Antimicrobial susceptibility testing: A review of general principles and contemporary practices. Clinical Infectious Diseases: An Official Publication of the Infectious Diseases Society of America, 49(11), 1749-1755. https://doi.org/10.1086/647952 
545 Kiehlbauch, J. A., Hannett, G. E., Salfinger, M., Archinal, W., Monserrat, C., \& Carlyn, C. (2000).

546

Use of the National Committee for Clinical Laboratory Standards guidelines for disk diffusion susceptibility testing in New York state laboratories. Journal of Clinical Microbiology, 38(9), 3341-3348. https://doi.org/10.1128/JCM.38.9.3341-3348.2000

Labun, K., Montague, T. G., Gagnon, J. A., Thyme, S. B., \& Valen, E. (2016). CHOPCHOP v2: A web tool for the next generation of CRISPR genome engineering. Nucleic Acids Research, 44(W1), W272-W276. https://doi.org/10.1093/nar/gkw398

Labun, K., Montague, T. G., Krause, M., Torres Cleuren, Y. N., Tjeldnes, H., \& Valen, E. (2019). CHOPCHOP v3: Expanding the CRISPR web toolbox beyond genome editing. Nucleic Acids Research, 47(W1), W171-W174. https://doi.org/10.1093/nar/gkz365

Liu, X., Homma, A., Sayadi, J., Yang, S., Ohashi, J., \& Takumi, T. (2016). Sequence features associated with the cleavage efficiency of CRISPR/Cas9 system. Scientific Reports, 6(1), 19675. https://doi.org/10.1038/srep19675

Lyden, A. (2019). In Vitro Transcription for dgRNA. https://doi.org/10.17504/protocols.io.3bpgimn

Quan, J., Langelier, C., Kuchta, A., Batson, J., Teyssier, N., Lyden, A., Caldera, S., McGeever, A., Dimitrov, B., King, R., Wilheim, J., Murphy, M., Ares, L. P., Travisano, K. A., Sit, R., Amato, R., Mumbengegwi, D. R., Smith, J. L., Bennett, A., ... Crawford, E. D. (2019). FLASH: A next-generation CRISPR diagnostic for multiplexed detection of antimicrobial resistance sequences. Nucleic Acids Research, 47(14), e83. https://doi.org/10.1093/nar/gkz418

R Core Team (2017). R: A language and environment for statistical computing. R Foundation for Statistical Computing, Vienna, Austria. URL https://www.R-project.org/

Seemann, T. (2014). Prokka: Rapid prokaryotic genome annotation. Bioinformatics, 30(14), 2068-2069. https://doi.org/10.1093/bioinformatics/btu153

Stoddard, S. F., Smith, B. J., Hein, R., Roller, B. R. K., \& Schmidt, T. M. (2015). rrnDB: Improved tools for interpreting rRNA gene abundance in bacteria and archaea and a new foundation for future development. Nucleic Acids Research, 43(Database issue), 
574 Syal, K., Mo, M., Yu, H., Iriya, R., Jing, W., Guodong, S., Wang, S., Grys, T. E., Haydel, S. E., \& Tao, N. (2017). Current and emerging techniques for antibiotic susceptibility tests. Van Hamelsveld, S., Adewale, M. E., Kurenbach, B., Godsoe, W., Harding, J. S., Theranostics, 7(7), 1795-1805. https://doi.org/10.7150/thno.19217 Remus-Emsermann, M. N. P., \& Heinemann, J. A. (2019). Prevalence of antibiotic-resistant Escherichia coli isolated from urban and agricultural streams in Canterbury, New Zealand. FEMS Microbiology Letters, 366(8).

https://doi.org/10.1093/femsle/fnz104

Wick, R. R., Judd, L. M., Gorrie, C. L., \& Holt, K. E. (2017). Unicycler: Resolving bacterial genome assemblies from short and long sequencing reads. PLOS Computational Biology, 13(6), e1005595. https://doi.org/10.1371/journal.pcbi.1005595

Wilson, W. J., Strout, C. L., DeSantis, T. Z., Stilwell, J. L., Carrano, A. V., \& Andersen, G. L. (2002). Sequence-specific identification of 18 pathogenic microorganisms using microarray technology. Molecular and Cellular Probes, 16(2), 119-127. https://doi.org/10.1006/mcpr.2001.0397

Zumla, A., Al-Tawfiq, J. A., Enne, V. I., Kidd, M., Drosten, C., Breuer, J., Muller, M. A., Hui, D., Maeurer, M., Bates, M., Mwaba, P., Al-Hakeem, R., Gray, G., Gautret, P., Al-Rabeeah, A. A., Memish, Z. A., \& Gant, V. (2014). Rapid point of care diagnostic tests for viral and bacterial respiratory tract infections-Needs, advances, and future prospects. The Lancet Infectious Diseases, 14(11), 1123-1135. https://doi.org/10.1016/S1473-3099(14)70827-8 


\section{Supplementary figures}

Purify DNA from sample and dephosphorylate all DNA

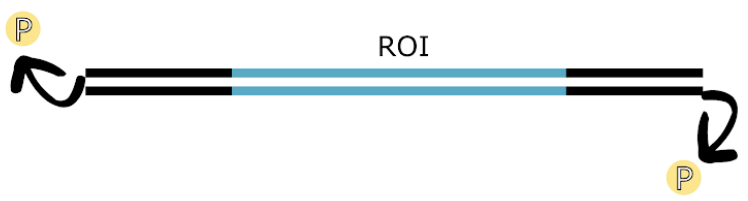

Form the Cas9-ribonucleoprotein complex

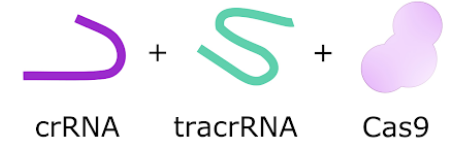

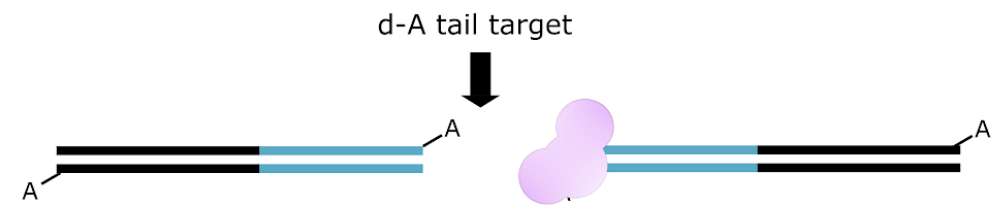

Ligate adapter and motor, add to flow cell, sequence
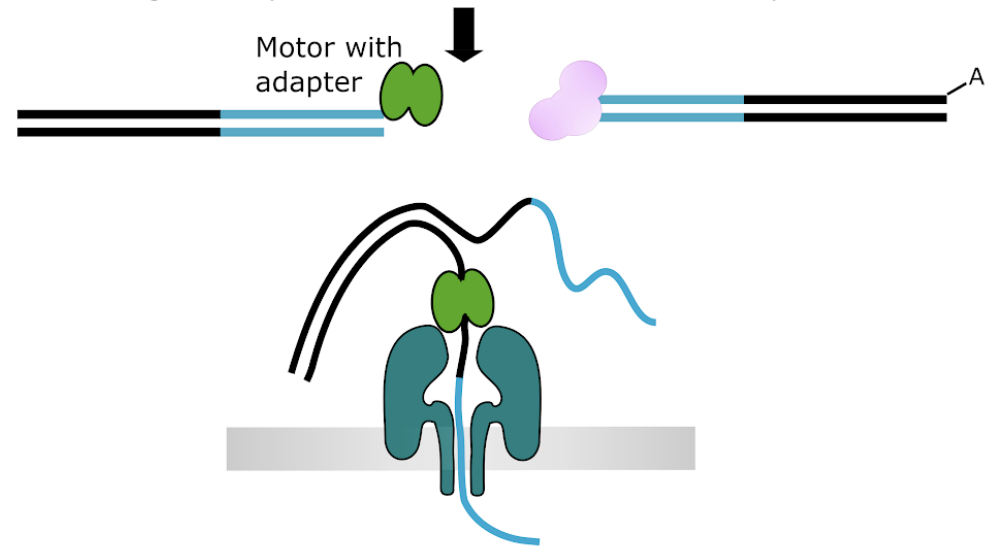

598 Figure S1. Illustration of the method for CRISPR-Cas9 enrichment and sequencing of targeted regions. First, genomic DNA is dephosphorylated. Ribonucleoprotein complexes (RNP) are formed from cas9, tracrRNA, and crRNA targeting the region of interest. Once cut, the ends of the target are $\mathrm{d}-\mathrm{A}$ tailed, and sequencing adapters are ligated. 
bioRxiv preprint doi: $h$ ttps://doi.org/10.1101/2020.09.30.320226; this version posted November 4, 2020. The copyright holder for this preprint (which was not certified by peer review) is the author/funder, who has granted bioRxiv a license to display the preprint in perpetuity. It is made available under aCC-BY 4.0 International license.
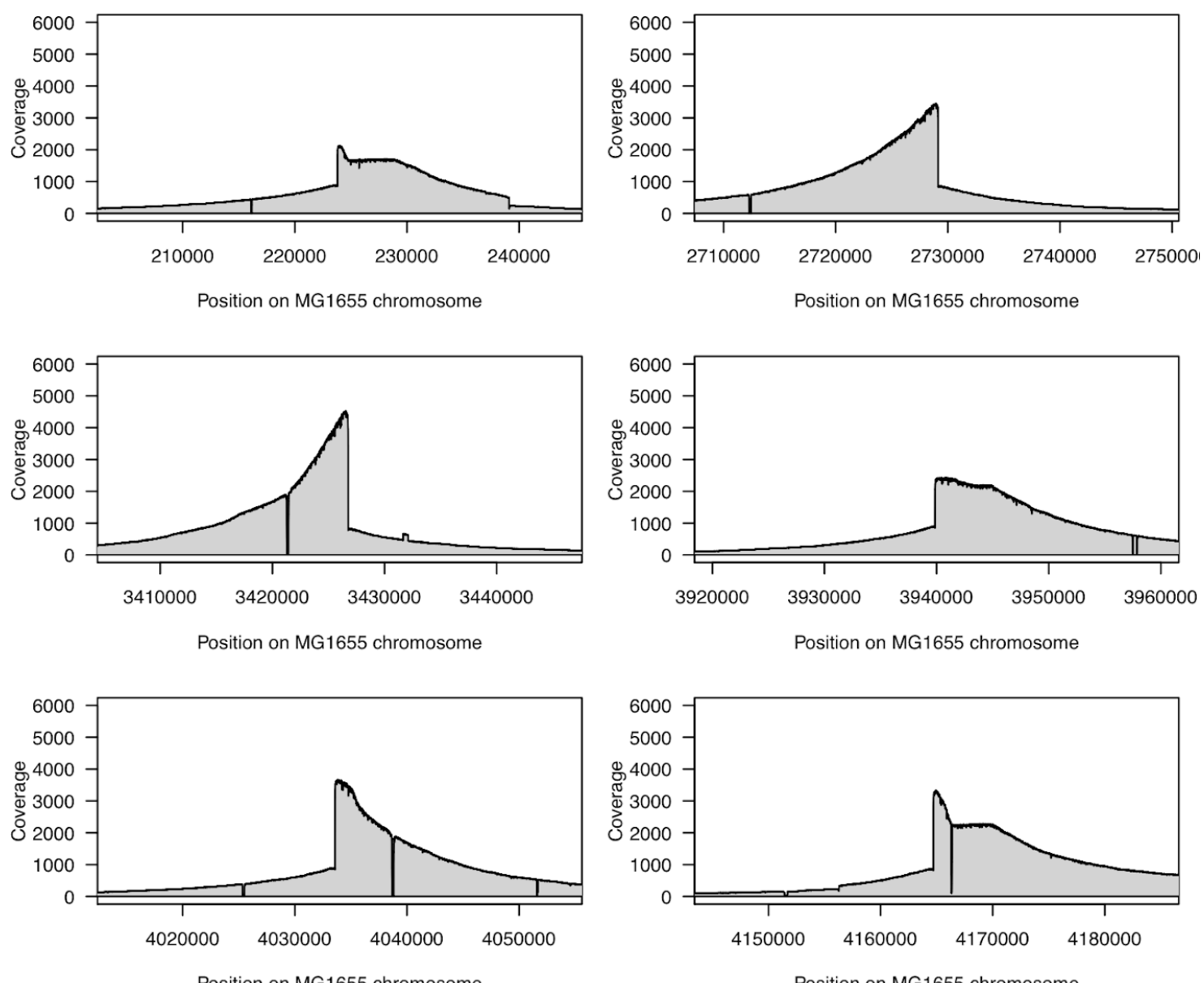

Position on MG1655 chromosome

Position on MG1655 chromosome

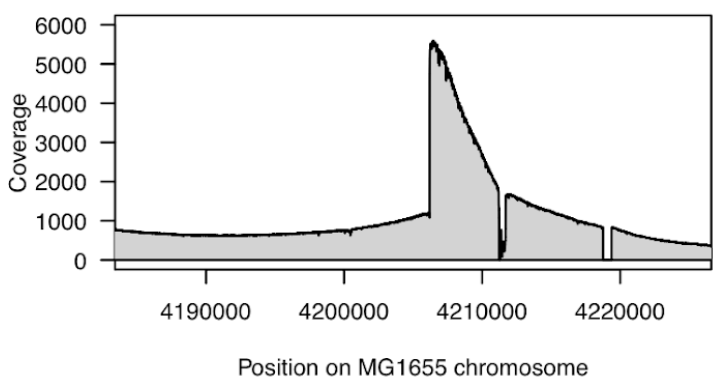

604 Figure S2. Small insertions and deletions at all 16S loci distinguish E. coli L3Cip3 from MG1655.

605 Each panel shows the coverage depth at one of the seven 16S operons in K12 when mapping reads from

606 a Bac-PULCE run targeting L3Cip3 16S with a crRNA. Small insertions and deletions are readily

607 apparent and allow strains to be distinguished when using long reads.

608 
bioRxiv preprint doi: https://doi. org/10.1101/2020.09.30.320226; this version posted November 4, 2020. The copyright holder for this preprint (which was not certified by peer review) is the author/funder, who has granted bioRxiv a license to display the preprint in perpetuity. It is made available under aCC-BY 4.0 International license.

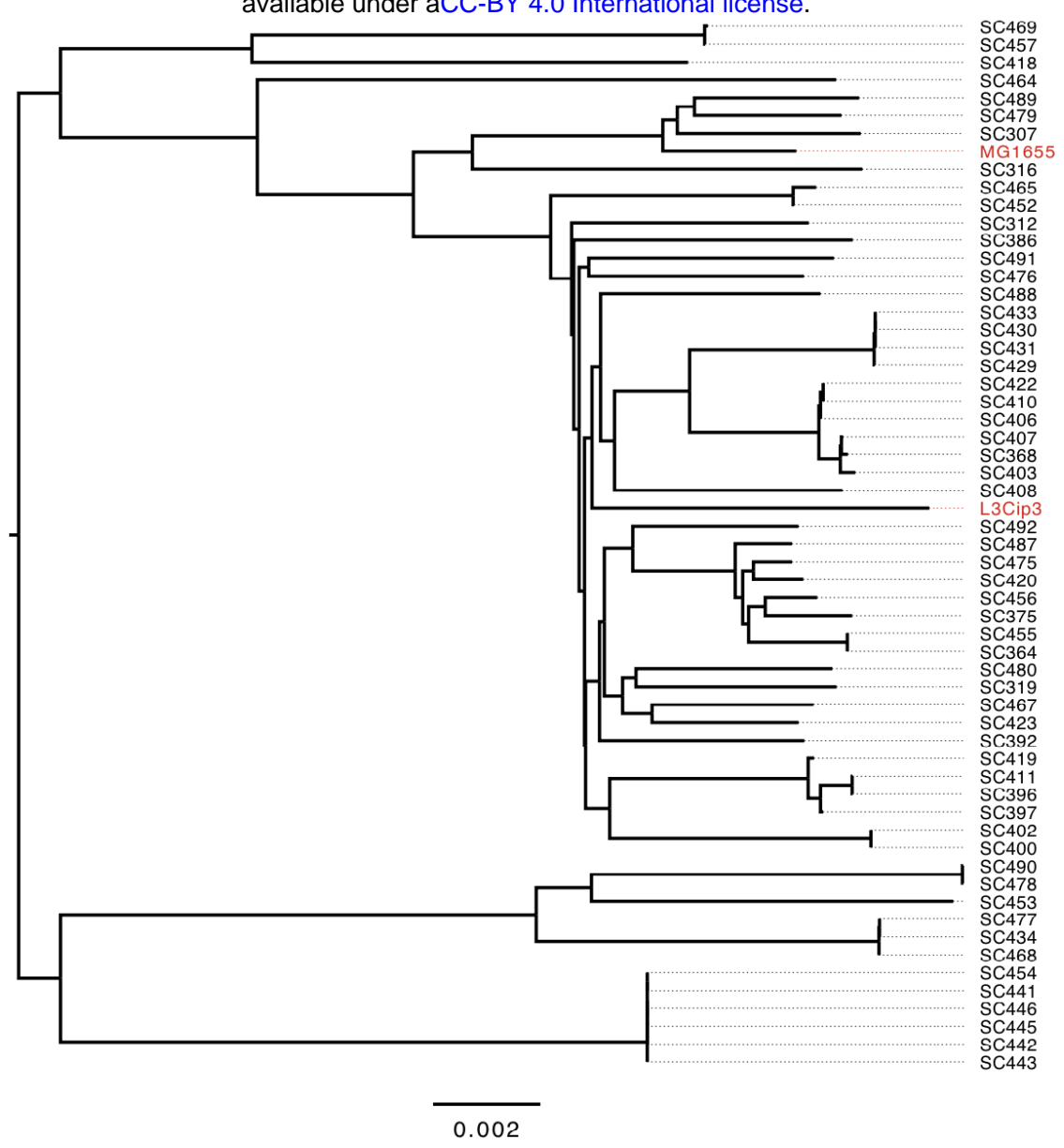

609

610 Figure S3. Phylogeny of strains used to test accuracy of strain classification using gnd and 16S

611 Bac-PULCE reads. The phylogeny was constructed from whole genome sequences (Breckell \& Silander, 612 2020) using REALPHY (Bertels et al., 2014). E. coli K12 MG1655 and L3Cip3 are highlighted in red. This 613 figure was constructed using FigTree. 

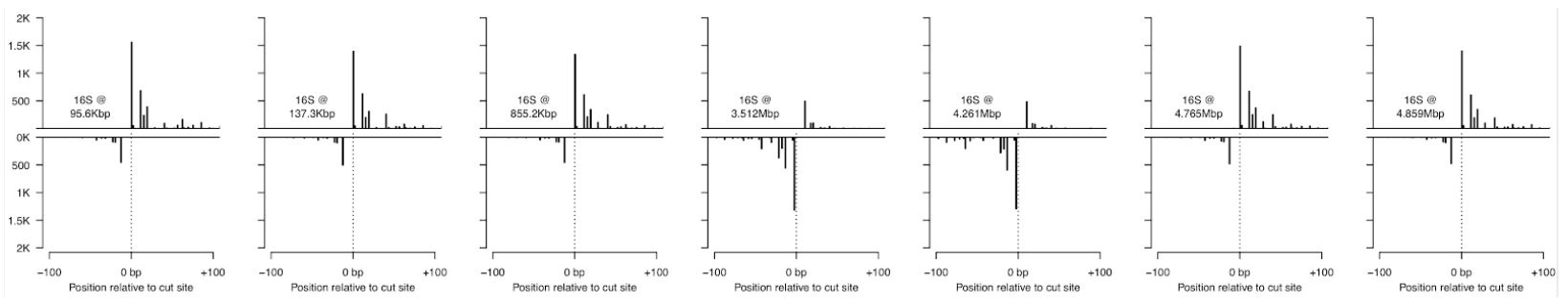

617 Figure S4. crRNA Binding and cutting efficiency and directionality bias are nearly identical at 16S

618 rRNA regions. The seven plots indicate the number of reads starting near each crRNA cut site. Lines above the axis indicate reads starting on the top strand; lines below begin on the bottom strand. The plots are shown in the order of cut sites, with the locations of the cut sites indicated on each plot. There is very little difference in cutting efficiency or directionality at each site, indicated by the similarity of the sequence start profiles. Reads on the fourth and fifth plots appear primarily on the bottom strand as these operons are organised in the opposite direction compared to the other $16 \mathrm{~S}$ operons. 